\title{
Phase I study of the anti-IGF1R antibody cixutumumab with everolimus and octreotide in advanced well-differentiated neuroendocrine tumors
}

\author{
Arvind Dasari, Alexandria Phan ${ }^{\dagger}$, Sanjay Gupta, Asif Rashid, Sai-Ching Jim Yeung, \\ Kenneth Hess, Helen Chen', Emily Tarco, Huiqin Chen, Caimiao Wei, Kim Anh-Do, \\ Daniel Halperin, Funda Meric-Bernstam and James Yao \\ Department of Gastrointestinal Medical Oncology, The University of Texas M.D. Anderson Cancer Center, \\ 1515 Holcombe Street, Unit 426, Houston, Texas 77030, USA \\ ${ }^{1}$ National Cancer Institute, Rockville, Maryland, USA \\ ${ }^{+} A$ Phan is now at The Methodist Hospital, Houston, Texas, USA
}

Correspondence should be addressed to $J$ Yao

Email

jyao@mdanderson.org

\begin{abstract}
Preclinical data suggest multiple roles for the IGF1 receptor (IGF1R) in neuroendocrine tumors (NETs), including mediating resistance to mammalian target of rapamycin (mTOR) inhibitors. Everolimus, an oral mTOR inhibitor, and octreotide long-acting repeatable (LAR) are approved for subgroups of well-differentiated NET. The primary objective of the present study was to establish the safety and recommended phase II dose (RP2D) of cixutumumab, a monoclonal antibody (MAB) against IGF1R, with everolimus and octreotide LAR. Patients with well-differentiated NET were treated with $10 \mathrm{mg}$ everolimus p.o. daily, $20 \mathrm{mg}$ octreotide LAR i.m. every 21 days, and escalating doses of cixutumumab. An expansion cohort was enrolled at RP2D. Correlative studies included the evaluation of mTOR pathway inhibition in paired tumor biopsies and the effects of this combination on metabolism via indirect calorimetry. Nineteen patients with progressive disease were enrolled, including nine to the expansion portion. Two patients had dose-limiting toxicities of grade 3 mucositis at $15 \mathrm{mg} / \mathrm{kg}$ cixutumumab. Long-term tolerance at RP2D was problematic, and the most common $\geq$ grade 3 adverse event was fatigue. One patient with metastatic insulinoma had a confirmed partial response, whereas 17 had stable disease. The median progression-free survival was 43.6 weeks, and the median overall survival was 25.5 months. The RP2D of this combination per the predefined study protocol of $10 \mathrm{mg} / \mathrm{kg}$ cixutumumab i.v., $20 \mathrm{mg}$ octreotide LAR i.m. every 21 days plus $10 \mathrm{mg}$ everolimus p.o. daily is associated with non-dose-limiting toxicities that limit long-term tolerance. Although a signal of activity was noted in the present study, this will need to be reconciled with limited tolerance of the combination and data from larger studies of anti-IGF1R MABs in NET that have been disappointing.
\end{abstract}
Key Words
- IGF
- neuroendocrine tumors
- intracellular signaling
- carcinoids

Endocrine-Related Cancer (2015) 22, 431-441
(C) 2015 Society for Endocrinology Printed in Great Britain
Published by Bioscientifica Ltd 


\section{Introduction}

The incidence of neuroendocrine tumors (NETs) has increased from 1.09 cases/100 000 in 1973 to 5.25/ 100000 in 2004 (Yao et al. 2008). Although they are often slow growing, these cancers are nonetheless incurable and lethal when advanced. However, treatment options continue to be limited, especially for carcinoid tumors. Mutations in the mammalian target of rapamycin (mTOR) pathway have been reported in $14 \%$ of pancreatic NETs (pNETs), and such abnormalities have also been associated with more aggressive tumors (Meric-Bernstam \& GonzalezAngulo 2009, Jiao et al. 2011). The phase III randomized, placebo-controlled RADIANT-3 study showed significant improvement in progression-free survival (PFS) with everolimus, an oral inhibitor of mTOR in patients with pNETs (Yao et al. 2011). Another placebo-controlled phase III study, RADIANT-2, randomized patients with advanced NETs associated with carcinoid syndrome to octreotide long-acting repeatable (LAR) in combination with placebo or everolimus and showed improved PFS in the everolimus arm, although this did not meet the predefined threshold for significance (Pavel et al. 2011). Insulin-like growth factor 1 receptors (IGF1Rs) and ligands are known to be expressed at higher concentrations in NETs than in normal tissue (Wulbrand et al. 2000). Studies in carcinoid cell lines show that IGF1 is a major regulator of neuroendocrine secretion and growth (von Wichert et al. 2000). Other in vitro studies have shown that blockade of IGF1R results in inhibition of NET cell growth and the induction of apoptosis (Hopfner et al. 2006). Mouse models of pNETs suggest a role for IGF2 in tumorigenesis (Christofori et al. 1995). Finally, other preclinical studies have suggested that IGF1 protects cancer cells from rapamycin-induced cell death and that inhibition of IGF1R prevents rapamycin-induced AKT activation and sensitizes tumor cells to mTOR inhibitors, which thus provides a rationale for combined blockade of these pathways (Thimmaiah et al. 2003, O'Reilly et al. 2006).

The goals of the present phase I study were to establish the safety and recommended phase II dose (RP2D) of everolimus and octreotide LAR in combination with escalating doses of the anti-IGF1R MAB and to evaluate this combination for preliminary evidence of activity in patients with advanced low- to intermediate-grade NETs.

\section{Patients and methods}

\section{Eligibility criteria}

Eligible patients had progressive advanced or metastatic low- or intermediate-grade neuroendocrine cancers (carcinoid of any site or pNET) refractory to or without standard therapy options and evaluable or measurable disease by Response Evaluation Criteria in Solid Tumors (RECIST) (Therasse et al. 2000) and Eastern Cooperative Oncology Group performance status of $0-1$. Patients may have received prior therapy (except mTOR inhibitors or agents that targeted IGF1R) with no limitation on the number of prior regimens. Patients also had to be aged 18 years or older with adequate hematopoietic, hepatic, and kidney function, fasting serum glucose $\leq 132 \mathrm{mg} / \mathrm{dl}$, fasting serum cholesterol $\leq 300 \mathrm{mg} / \mathrm{dl}$, and fasting triglycerides $\leq 375 \mathrm{mg} / \mathrm{dl}$. Exclusion criteria included: women who were pregnant or nursing; treatment with radiotherapy or chemotherapy within the past 4 weeks; major surgery within the past 3 weeks; known CNS metastasis requiring anticonvulsants or steroids; patients on therapeutic anticoagulation not on stable dosages; uncontrolled severe cardiac disease or diabetes mellitus; any medical condition that would impair the administration of an oral medication; prior mTOR and/or IGF1R therapies; and uncontrolled serious medical or psychiatric illness.

The protocol was approved by the Institutional Review Board at the M.D. Anderson Cancer Center, and written informed consent was obtained for all patients before study-related procedures were performed in accordance with federal and institutional guidelines.

\section{Study design}

The present study was a single-institution, single-arm, inter-patient, dose-escalation study with a dose-expansion cohort of nine patients enrolled at the recommended dose. The total duration of treatment was 12 months.

\section{Drug administration and dose-escalation procedures}

Cixutumumab was supplied by the Division of Cancer Treatment and Diagnosis of the National Cancer Institute (Bethesda, MD, USA) as single-use vials, and weight-based dose was administered i.v. over 90 min every 21 days. Everolimus was supplied by Novartis Pharmaceuticals and was orally administered to fasting patients on a once daily continuous dosing schedule. Keeping the doses of everolimus (10 mg p.o. daily) and octreotide LAR (20 mg i.m. every 21 days) constant, cixutumumab was evaluated at escalating doses of 10 and $15 \mathrm{mg} / \mathrm{kg}$ every 21 days for a 21-day cycle. Octreotide LAR was administered every

Published by Bioscientifica Ltd. 
21 days rather than the standard practice of every 28 days to fit with the study schedule for patients' convenience.

For the dose-escalation portion of the study, patients were enrolled sequentially into escalating dosing cohorts of six patients each rather than the traditional $3+3$ design, because at the beginning of the study, it was unclear if the maximum tolerated dose (MTD) would be reached. Therefore, larger cohorts were chosen in order to better characterize pharmacodynamics (PD) and the RP2D. Intrapatient dose escalation was not permitted, and once each sequential cohort was enrolled, accrual was held until all of the patients in that cohort had completed one cycle of therapy. Dose-limiting toxicity (DLT) was defined as: any $\geq$ grade 4 hematological toxicity, grade 3 neutropenia, or thrombocytopenia that did not resolve to either grades 0 or 1 following dose interruption for $>7$ days; $\geq$ grade 3 non-hematologic toxicity (except $\geq$ grade 3 hyperglycemia, hyperlipidemia that could be controlled with appropriate medical management within 3 weeks, and $\geq$ grade 3 nausea or vomiting that could be controlled with medication within 7 days); or any toxicity that led to $>14$ days of treatment delay that occurred during the first cycle with definite, possible, or probable attribution to cixutumumab and/or everolimus. If one or no patients in any cohort of six patients developed DLT, dose escalation was permitted. If two or more patients in any expanded cohort developed DLT, dose escalation was stopped, and the cohort immediately below was deemed to be RP2D.

For patients with $\geq$ grade 3 treatment-related toxicities, dosing of the drug was held until resolution to $\leq 1$ and restarted at one dose-level reduction for hematological toxicities and per physician's discretion for other transient grade 4 toxicities. For IMC-A12, doses were reduced to the next lower level in increments of $5 \mathrm{mg} / \mathrm{kg}$ i.v. every 21 days up to $5 \mathrm{mg} / \mathrm{kg}$ i.v. every 21 days. For everolimus, the dose was successively reduced from 10 to $5 \mathrm{mg}$ p.o. daily to $5 \mathrm{mg}$ p.o. every other day. If further reductions of either drug were required or if patients required a dose delay of $>21$ days, therapy with that drug was discontinued permanently.

\section{Clinical evaluation and safety assessment}

Before study entry, patients underwent clinical history and physical examination, performance status assessment, vital signs measurement, complete blood count (СBC), chemistries, coagulation parameters, urinalysis, serum pregnancy test, tumor markers assessment, HbA1c measurement, fasting total cholesterol and triglycerides measurement, electrocardiogram (ECG), indirect calorimetry, and baseline tumor measurements. During the study, patients underwent evaluation, including clinical history and physical examination, vital signs measurement, СBC, lipid panel assessment, and chemistries every cycle. Urinalysis was repeated each cycle. Given prior reports of hearing loss in patients treated with IGF1R MABs, audiometry testing with comprehensive audiometry and tympanometry was performed at baseline and at the end of the study. Adverse events (AEs) were classified/graded weekly according to the Common Terminology Criteria of Adverse Events version 4.0. Response was assessed every three cycles and evaluated by RECIST version 1 as in other major NET trials (Therasse et al. 2000). All patients who received at least one treatment were considered evaluable for toxicity, and those who had received at least one complete cycle of therapy and had their disease reevaluated were considered evaluable for response.

\section{PD analysis}

Paired pre- (within 4 weeks of initiating therapy) and posttreatment biopsies ( $24 \mathrm{~h}$ after day 1 administration of cixutumumab and everolimus during cycle 2) were obtained and were frozen immediately for reverse phase protein array (RPPA) or were formalin-fixed and paraffinembedded for immunohistochemistry (IHC).

\section{Immunohistochemistry}

IHC was performed on pre-treatment and post-treatment core biopsies with the following antibodies as previously reported (Akcakanat et al. 2008, Meric-Bernstam et al. 2014): pAKT $S 473$ (Cell Signaling, Danvers, MA, USA), pS6 S235/236 (Cell Signaling), pS6 S240/244 (Cell Signaling), and p4E-BP1 T70 (Cell Signaling). Ki-67 immunostaining was performed in the M.D. Anderson IHC Core Laboratory with a Ki-67 antibody (Dako Carpinteria, CA, USA). Positive and negative controls were included for all assays. Immunoreactivity scores (IRS) were calculated as \% staining multiplied by intensity.

\section{Reverse phase protein array}

Protein lysate was extracted and RPPA was performed in the M.D. Anderson Cancer Center Functional Proteomics Core Facility as described previously (Meric-Bernstam et al. 2012). Samples were resuspended in three volumes of RPPA lysis buffer (Hennessy et al. 2010) that had already been mixed with one volume of $4 \times$ SDS sample buffer. Samples were sonicated using a microtip probe

Published by Bioscientifica Ltd 
(Fisher Scientific, Waltham, MA, USA), and protein concentrations were determined using a BCA Kit (Pierce, Rockford, IL, USA). 2-Mercaptoethanol was added (10\% $(\mathrm{v} / \mathrm{v})$ of $4 \times$ SDS sample buffer), and final protein concentration was adjusted to $3 \mu \mathrm{g} / \mu \mathrm{l}$. Samples were probed with antibodies validated for RPPA that were enriched for components of the PI3K/AKT/mTOR pathway. A total of 161 unique proteins (including phosphoproteins) were evaluated. The list of antibodies is included in Supplementary Table 1, see section on supplementary data given at the end of this article. Analyses of the log-transformed protein levels were performed.

\section{Indirect calorimetry}

Given the known effects of the IGF and mTOR signaling pathways on metabolism, indirect calorimetry was obtained at baseline and on cycle 2 day 1 using a handheld indirect calorimeter in a rested and fasting state.

\section{Statistical analyses}

Descriptive statistics were used for baseline characteristics, safety assessments, and exploratory assessments, including PFS, overall survival, and tumor response.

The RPPA spot signal intensity data from MicroVigene was processed using the $\mathrm{R}$ package SuperCurve version 1.4 .3 (http://bioinformatics.mdanderson.org/OOMPA) as previously described (Meric-Bernstam et al. 2012). The modified $z$ scores of five important mTOR pathway biomarkers were calculated and used to compute the composite mTOR pathway activity score. The composite mTOR pathway activity score was defined as the sum of the modified $z$ scores of phosphoprotein of $4 \mathrm{E}-\mathrm{BP} 1, \mathrm{~S} 6 \mathrm{~K}$, and S6 (i.e., score $=$ pS6 $240 / 244+$ pS6 S235/236+pS6K T389+p4E-BP1 S65+p4E-BP1 T37/46). The modified $z$ score proposed by Iglewicz \& Hoaglin (1993) was calculated based on the median of expressions and absolute deviation about the median. A linear mixed model was used to compare the mTOR activity scores and individual protein expression between pre- and posttreatment. Regulation of mTOR pathway biomarkers and mTOR pathway activity was performed as planned analysis $(P \leq 0.05)$, and functional proteomics profile was performed as exploratory analysis. To account for multiple testing, we estimated the false discovery rate (FDR) of the model using the Benjamini-Hochberg method, with the FDR-adjusted $P$ value of $q<0.3$ being significant.

Spearman's rank correlation $(\rho)$ was used to assess the associations between protein expression at pre-treatment and on-treatment as well as the change and the best response in \% tumor size change.

Univariate cox proportional hazards model was fitted to assess the association between the $z$ score of biomarker expression at pre-treatment and on-treatment as well as the PD $z$ score change $(z \mathrm{PD} \Delta=$ on-treatment $z$ scorepre-treatment $z$ score) and PFS. Statistical significance was measured using the Wald test. Again, we adjusted for multiple testing as described earlier in this section.

The exact Wilcoxon's signed rank test was applied to compare the immunostaining of the pre-treatment and on-treatment biopsies. For markers that had a range of intensity, the highest intensity was used for analysis.

Changes in indirect calorimetry from baseline to cycle 2 were tested using the Wilcoxon' signed rank test. NCT trial identifier: NCT01204476.

\section{Results}

\section{Patient demographics and treatment}

Nineteen patients, ten in the dose-escalation portion and nine in the MTD expansion portion, were enrolled from November 2010 to May 2012 (Table 1). None of the patients had multiple endocrine neoplasia or other hereditary syndromes associated with NETs. All of the patients had progression of the disease before enrollment on trial. A total of 207 cycles were administered. The median number of cycles per patient was ten (range 3-19). Reasons for study discontinuation included disease progression in eight patients, completion of study in four patients, toxicities in four patients, and withdrawal of consent in two patients. One patient was taken off the study per the discretion of his treating physician because of complications from carcinoid heart disease.

\section{Dose escalation and determination of MTD}

In the $10 \mathrm{mg} / \mathrm{kg}$ cixutumumab cohort, six patients were treated without any DLTs. However, at the next cohort of $15 \mathrm{mg} / \mathrm{kg}$ cixutumumab, two of four patients experienced DLTs with both developing grade 3 mucositis. Further dose escalation was hence stopped, and the RP2D was deemed to be $10 \mathrm{mg} / \mathrm{m}^{2}$ cixutumumab, $10 \mathrm{mg}$ everolimus p.o. daily, and $20 \mathrm{mg}$ octreotide LAR i.m. every 21 days (i.e., the previous dose level). An additional nine patients were enrolled to further evaluate tolerability and preliminary antitumor activity, and no further DLTs were noted.

Published by Bioscientifica Ltd 
Table 1 Baseline demographics and patient characteristics

\begin{tabular}{|c|c|c|}
\hline \multirow[b]{2}{*}{ Characteristic } & \multicolumn{2}{|c|}{$n=19$} \\
\hline & $n$ & $\%$ \\
\hline \multicolumn{3}{|l|}{ Sex } \\
\hline Male & 10 & 53 \\
\hline Female & 9 & 47 \\
\hline \multicolumn{3}{|l|}{ Age (years) } \\
\hline Median & 66 & \\
\hline Range & $45-79$ & \\
\hline \multicolumn{3}{|l|}{ ECOG performance status } \\
\hline 0 & 15 & 34 \\
\hline 1 & 4 & 66 \\
\hline \multicolumn{3}{|c|}{ Previous lines of systemic therapies } \\
\hline Median & 1 & \\
\hline Range & $0-3$ & \\
\hline \multicolumn{3}{|l|}{ Previous therapy } \\
\hline Chemotherapy & 9 & 47 \\
\hline Radiotherapy & 4 & 21 \\
\hline Somatostatin analogue & 13 & 68 \\
\hline Radioembolization & 2 & 11 \\
\hline VEGF-targeting antibody & 3 & 16 \\
\hline Interferon therapy & 1 & 5 \\
\hline Surgery & 8 & 42 \\
\hline \multicolumn{3}{|l|}{ NET origin } \\
\hline Pancreatic & 4 & \\
\hline Small bowel & 6 & \\
\hline Lung & 4 & \\
\hline Kidney & 1 & \\
\hline Other/unknown & 4 & \\
\hline \multicolumn{3}{|l|}{ Functional status } \\
\hline Functional & 6 & \\
\hline Non-functional & 13 & \\
\hline \multicolumn{3}{|l|}{ Number of metastatic sites } \\
\hline 1 & 5 & \\
\hline 2 & 8 & \\
\hline $2+$ & 6 & \\
\hline \multicolumn{3}{|l|}{ Time from diagnosis } \\
\hline Median & 18 & \\
\hline Mean & 38 & \\
\hline \multicolumn{3}{|l|}{ Serum chromogranin levels } \\
\hline $2 \times-4 \times$ ULN & 3 & \\
\hline$>4 \times$ ULN & 10 & \\
\hline
\end{tabular}

ECOG, Eastern Cooperative Oncology Group; VEGF, vascular endothelial growth factor.

\section{Safety}

All 19 patients were evaluable for toxicities, and all had at least one toxicity of any grade during the study. The most frequently observed AEs seen in all of the courses were fatigue, hyperlipidemia, and weight loss (all with incidence rates of 74\%); other common AEs included altered taste (68\%), hyperglycemia, and mucositis (both $63 \%$ ). Eleven $(57 \%)$ patients had at least one $\geq$ grade 3 toxicity. The most common grade $3 \mathrm{AE}$ was fatigue (21\%); other common grade 3 AEs included mucositis, elevated liver tests, and electrolyte abnormalities (all 11\%). There were no grade $5 \mathrm{AEs}$, and one patient had grade 4 hyperglycemia.

Only one patient completed the entire study per protocol at RP2D. The mean number of cycles delivered at the full RP2D in the 15 patients treated at this level was 4 (median 3). Four patients were taken off the study because of toxicities, which included persistent grade 3 neutropenia $(n=1)$, fatigue $(n=3)$, and fatigue and weight loss $(n=1)$. Two patients withdrew consent, citing toxicities that included fatigue, nausea, and rash. Fifteen patients required dose reductions and/or interruptions because of toxicities. Everolimus was dose reduced in all 15 patients - most commonly because of thrombocytopenia $(n=5)$, fatigue $(n=4)$, and mucositis $(n=3)$. Of these 15 patients, eight required dose reduction of everolimus during or before cycle 2 . Other reasons included elevated creatinine, hyperglycemia, and altered mental status $(n=1$ each). One patient who elected to stop everolimus while on study stayed on cixutumumab and octreotide LAR until the end of protocol. Three patients had dose reduction in cixutumumab because of thrombocytopenia, altered mental status, and hyperglycemia ( $n=1$ each). Cixutumumab was discontinued in one patient because of grade 3 elevation in alkaline phosphatase. Octreotide LAR was not dose reduced in any patients, but it was discontinued in one patient because of bradycardia.

One patient developed worsening carcinoid heart disease and was taken off study per his physician's discretion, but it was thought to be unlikely that this was related to the study drugs. He underwent valve replacement and died several weeks later from postoperative complications.

Fifteen patients had baseline and end of study audiometry assessments; four patients had a grade 1 change in hearing sensitivity unilaterally, and one patient had bilateral changes (grades 1 and 2) that were not clinically significant and were identified on audiograms.

\section{Pharmacodynamic analyses}

All 19 patients underwent pre-treatment biopsies, and 15 had post-treatment biopsies. All pre-treatment and on-treatment H\&Es were reviewed, and patients without tumors on core biopsy samples had their samples excluded from IHC and RPPA analysis. The overall difference between pre- and post-treatment biopsies demonstrated statistically significant decline with treatment for S6 pS240/244 $(P=0.045)$ and S6 pS235/236 $(P=0.028)$, two proteins that are frequently assessed as downstream markers of mTOR signaling (Fig. 1A and B). The mTOR

Published by Bioscientifica Ltd 

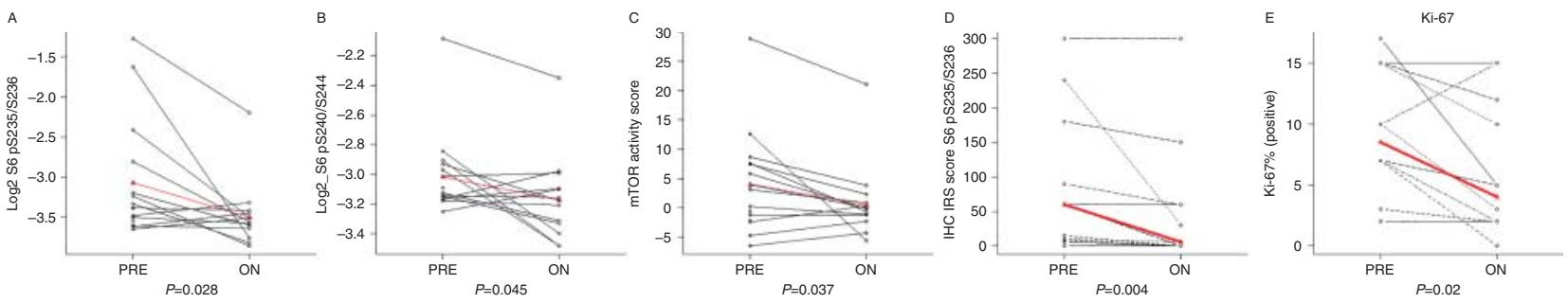

Figure 1

Pharmacodynamic changes in mTOR pathway activity with treatment: $S 6$ pS240/244 (A), S6 pS235/236 (B), mTOR pathway activity score (C), IRS S6

pS235/236 score (D), and Ki-67 (E). A full colour version of this figure is available at http://dx.doi.org/10.1530/ERC-15-0002.

activation score significantly decreased with treatment (adjusted mean pre-treatment 4.025 vs on-treatment $-0.41, P=0.037$; Fig. 1C). Thus, the RPPA analysis confirmed mTOR pathway inhibition by the treatment.

We next assessed whether baseline proteomics profile or PD changes were associated with best tumor response, which was represented as \% change in tumor size. Pretreatment expression of $\mathrm{ER} \alpha \mathrm{pS} 118$ was strongly inversely correlated with \% tumor size change (Supplementary Table 2, see section on supplementary data given at the end of this article). STAT3 pY705, SMAD3, RPTOR, PRAS40, pT246, and Annexin VII (ANXA7) were moderately inversely correlated with \% tumor size change, and four biomarkers (YAP pS127, VEGFR2, AKT pT308, and MEK1 pS217/221) were moderately positively correlated with \% tumor size change. After adjusting for multiple testing, ER $\alpha$ pS118, smad3, and STAT3 pY705 retained significance.

The treatment-associated decrease in MEK1 pS217/221 was strongly inversely correlated with \% tumor size change, whereas ANXA7 and ER $\alpha$ pS118 were strongly positively correlated with \% tumor size change. The PD changes of 15 additional proteins were moderately negatively correlated, and those of 23 proteins were

Table 2 All hematologic and non-hematologic adverse events ${ }^{a}$. Worst grade per patient in all cycles in at least $25 \%$ of all patients and/or $\geq$ grade $3(n=19)$

\begin{tabular}{l}
\hline Adverse event \\
\hline Total \\
Fatigue \\
Hyperlipidemia \\
Weight loss \\
Dysguesia \\
Hyperglycemia \\
Mucositis \\
Diarrhea \\
Epistaxis \\
Thrombocytopenia \\
Anorexia \\
Rash \\
Pain \\
Nail ridging \\
Neutropenia \\
Elevated AST, ALT, or alk phos \\
Infection \\
Electrolyte abnormalities \\
Anemia \\
Injury \\
Retinal detachment \\
Thromboembolism \\
Dehydration
\end{tabular}

\begin{tabular}{|c|c|}
\hline \multicolumn{2}{|c|}{ Any grade } \\
\hline No. of patients & $\%$ \\
\hline 19 & 100 \\
\hline 14 & 74 \\
\hline 14 & 74 \\
\hline 14 & 74 \\
\hline 13 & 68 \\
\hline 12 & 63 \\
\hline 12 & 63 \\
\hline 11 & 58 \\
\hline 10 & 53 \\
\hline 10 & 53 \\
\hline 9 & 47 \\
\hline 8 & 42 \\
\hline 6 & 32 \\
\hline 6 & 32 \\
\hline 5 & 26 \\
\hline 5 & 26 \\
\hline 3 & 16 \\
\hline 3 & 16 \\
\hline 2 & 11 \\
\hline 1 & 5 \\
\hline 1 & 5 \\
\hline 1 & 5 \\
\hline 1 & 5 \\
\hline
\end{tabular}

\begin{tabular}{c} 
Grad \\
\hline No. of patients \\
\hline 10 \\
4 \\
0 \\
0 \\
0 \\
1 \\
2 \\
0 \\
0 \\
0 \\
1 \\
0 \\
0 \\
0 \\
1 \\
2 \\
1 \\
2 \\
1 \\
1 \\
1 \\
1 \\
1
\end{tabular}

\begin{tabular}{|c|c|}
\hline \multicolumn{2}{|c|}{ Grade 4} \\
\hline No. of patients & $\%$ \\
\hline 1 & 5 \\
\hline 0 & 0 \\
\hline 0 & 0 \\
\hline 0 & 0 \\
\hline 0 & 0 \\
\hline 1 & 5 \\
\hline 0 & 0 \\
\hline 0 & 0 \\
\hline 0 & 0 \\
\hline 0 & 0 \\
\hline 0 & 0 \\
\hline 0 & 0 \\
\hline 0 & 0 \\
\hline 0 & 0 \\
\hline 0 & 0 \\
\hline 0 & 0 \\
\hline 0 & 0 \\
\hline 0 & 0 \\
\hline 0 & 0 \\
\hline 0 & 0 \\
\hline 0 & 0 \\
\hline 0 & 0 \\
\hline 0 & 0 \\
\hline
\end{tabular}

ALT, alanine aminotransferase; AST, aspartate aminotransferase.

${ }^{a}$ Graded per the National Cancer Institute Common Terminology Criteria for Adverse Events version 3.

http://erc.endocrinology-journals.org DOI: $10.1530 /$ ERC-15-0002
(C) 2015 Society for Endocrinology Printed in Great Britain
Published by Bioscientifica Ltd. 
moderately positively correlated with \% tumor size change. After adjusting for multiple-tumor testing, 25 positively and 14 negatively correlated markers remained significant at FDR $<0.3$ (Supplementary Table 3).

A higher expression of AKT pT308 $z$ score on RPPA at pre-treatment was associated with worse PFS (hazard ratio $(\mathrm{HR})=2.379, P=0.011)$. The increase in $4 \mathrm{E}-\mathrm{BP} 1 \mathrm{pT} 37 / \mathrm{T} 46$ expression on-treatment was marginally significantly associated with worse PFS $(\mathrm{HR}=1.995, P=0.081$, Table 2). Greater increases in AKT pT308 and p70S6K pT389 expression between pre-treatment and on-treatment biopsies were significantly or marginally significantly associated with better PFS (AKT pT308: HR= $0.107, P=0.006$ and p70S6K pT389: $\mathrm{HR}=0.554, P=0.084$ ). Based on Supplementary Table 4, see section on supplementary data given at the end of this article, increased PD change in 15 proteins that had HRs of $<1$ was associated with a decreased risk of PFS. Increased PD $z$ score change in 21 proteins that had HRs of $>1$ were associated with an increased risk of PFS. All of these 36 biomarkers were significant at FDR $<0.3$.

IHC data was performed for five biomarkers (pAKT, Ki-67, p4E-BP1, pS6 235/236, and pS6 240/244). Thirteen patients had matched pre- and on-treatment IHC values that were evaluable for pAKT, pS6 235/236, and pS6 240/244; meanwhile, 12 patients had matched pre- and on-treatment IHC values of Ki-67 and p4E-BP1 T70. Treatment was associated with a statistically significant decrease in pS6 S235/236 IRS $(P=0.0063)$ and Ki-67 $(P=0.02)$. The pS6 S235/236, pS6 S240/244 immunostaining, and Ki-67 showed more robust inhibition in the patient who had a partial response as compared to another patient who experienced progressive disease (Fig. 2).

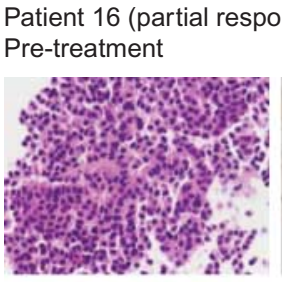

$H \& E$

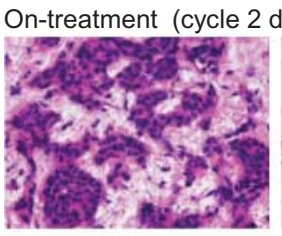

$H \& E$

pAkt (Ser473)

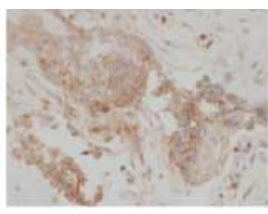

$1+$

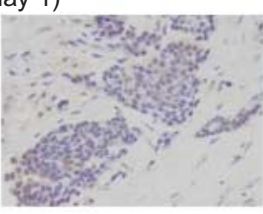

$0-1+$

Patient 19 (progressive disease) Pre-treatment

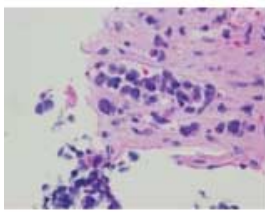

$H \& E$

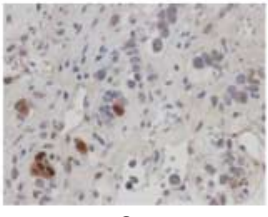

$2+$

On-treatment (cycle 2 day 1$)$

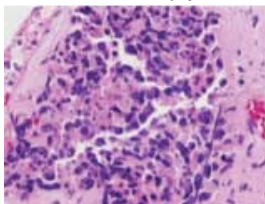

$H \& E$

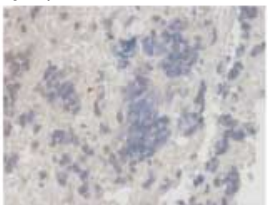

$0-1+$

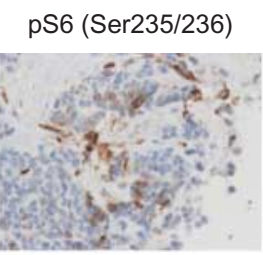

$3+$

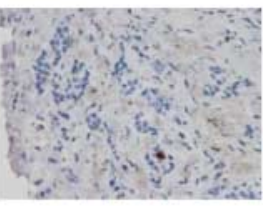

0

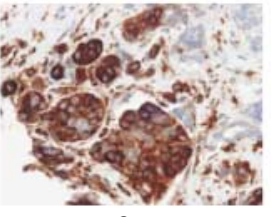

$3+$

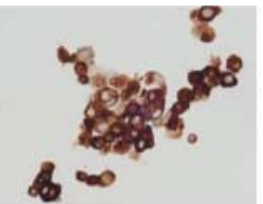

$3+$
pS6 (Ser240/244)

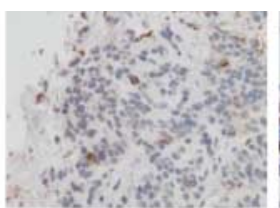

$3+$

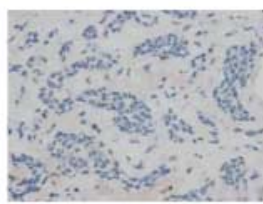

0

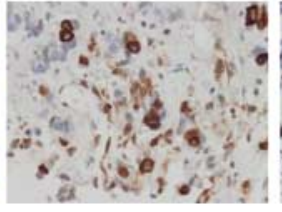

$3+$

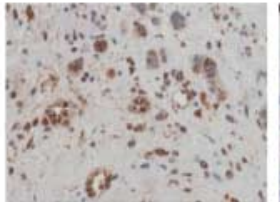

$3+$
Ki67

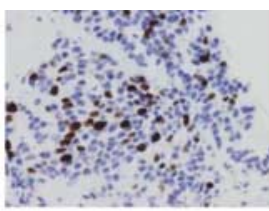

$17 \%$

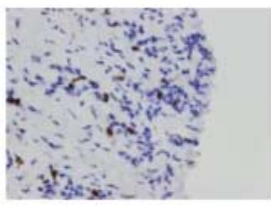

$5 \%$

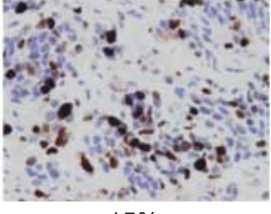

$15 \%$

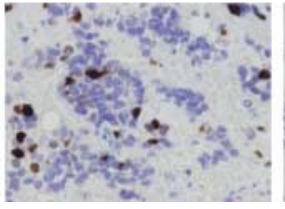

$10 \%$
p4EBP1 (Thr70)

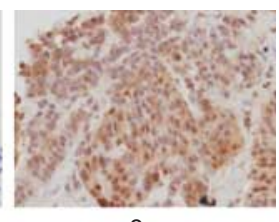

$3+$

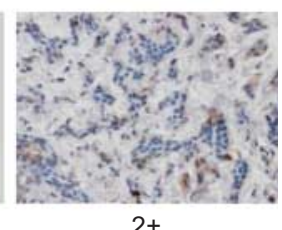

$2+$

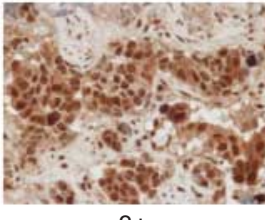

$3+$

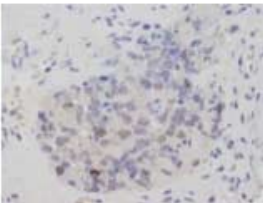

$0-1+$

Figure 2

IHC changes in mTOR pathway components and Ki-67 with treatment in responders vs non-responders. A full colour version of this figure is available at http://dx.doi.org/10.1530/ERC-15-0002.

http://erc.endocrinology-journals.org
DOI: $10.1530 /$ ERC-15-0002
(C) 2015 Society for Endocrinology Printed in Great Britain
Published by Bioscientifica Ltd 


\section{Metabolic analysis}

Fourteen patients had paired indirect calorimetry results at baseline and cycle 2 day 1 . Paired results were not obtained in the rest of the patients because they were either not fasting at the time of testing or they were not on treatment, and differences in mean values were calculated by paired $t$-test. The mean basal oxygen consumption $\left(\mathrm{VO}_{2}\right)$ was $216.7 \mathrm{l} / \mathrm{min}$ and the mean basal resting metabolic rate (RMR) was $1517.9 \mathrm{kcal} /$ day. The corresponding values at cycle 2 day 1 were significantly reduced to $190.2 \mathrm{ml} / \mathrm{min}$ for $\mathrm{VO}_{2}(P=0.001)$ and $1319.2 \mathrm{kcal} / \mathrm{day}$ for RMR $(P<0.001)$ (Fig. 3).

\section{Antitumor activity}

All 19 patients were eligible for response evaluation. There were no complete responses. One patient with metastatic insulinoma secreting proinsulin had a confirmed partial response. Before initiating therapy on the present trial, the patient's clinical course was marked by recurrent episodes of hypoglycemia, as detected by a continuous glucose monitoring system and also significant weight gain as a result of hormonal disturbances. However, within two cycles of therapy, he no longer had hypoglycemia and in fact required therapy with metformin for hyperglycemia. He was also able to lose a significant amount of weight, which is suggestive of normalization of his anabolic hormones, although his hyperglycemia also could have been an adverse effect of one or more of the study drugs. Seventeen patients had stable disease as best response, with a reduction in RECIST tumor measurements that

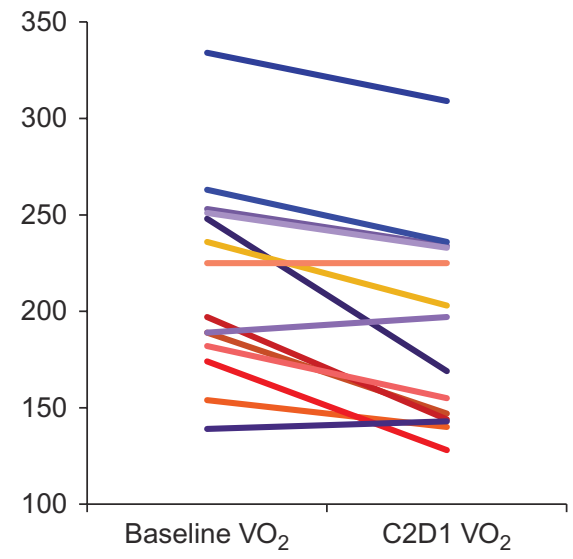

Figure 3

Changes in $\mathrm{VO}_{2}$ with treatment as measured by indirect calorimetry. A full colour version of this figure is available at http://dx.doi.org/10.1530/ ERC-15-0002. ranged from 1 to $22 \%$. Four patients were on study for the entire duration of 12 months. Median PFS was 43.6 weeks (range 9-132 weeks; 95\% CI 39.8-47.3 weeks). The median overall survival was 25.5 months (95\% CI 19.2-31.7 months). The median number of cycles of treatment was 10 (range 3-19). There were four patients with pNETs on the trial, including the patient with insulinoma described earlier in this section. The other three patients with pNETs had stable disease ranging from 20 to 44 weeks (Fig. 4).

\section{Discussion}

The primary objective of the present phase I study was to establish the RP2D of the combination of cixutumumab, everolimus, and octreotide LAR based on the observation of DLTs during the first cycle and was deemed to be $10 \mathrm{mg} / \mathrm{kg}$ cixutumumab, $10 \mathrm{mg}$ everolimus p.o. daily, and $20 \mathrm{mg}$ octreotide LAR i.m. every 21 days. The maximum dose of octreotide LAR was limited to $20 \mathrm{mg}$ i.m., as mandated by protocol. Although no further DLTs were noted, only one patient finished the study per protocol; the rest experienced significant toxicities, including most commonly fatigue, mucositis, and/or myelosuppression. All 15 of these patients required dose reduction of everolimus, and three patients required dose reduction of cixutumumab. The most common AEs noted with everolimus included stomatitis, rash, diarrhea, and fatigue, and the most common $\geq$ grade 3 AEs were stomatitis, myelosuppression, and hyperglycemia (Yao et al. 2011). In the phase II study of ganitumab, a human MAB against IGF1R in NET, the most common AEs included fatigue, hyperglycemia, and nausea, whereas the most common $\geq$ grade 3 AEs were myelosuppression and hyperglycemia (Strosberg et al. 2013). These overlapping toxicity profiles perhaps explain the poor long-term tolerance at RP2D. This underscores the need to look beyond MTD to establish long-term tolerability of novel therapies in patients with diseases that have an indolent course, such as NET.

In a preplanned analysis, changes in mTOR pathway biomarkers and mTOR pathway activity at cycle 2 day 1 as compared to baseline were evaluated. As evaluated by RPPA, the mTOR activity score significantly decreased with treatment, as did levels of downstream markers of the mTOR pathway, which suggested inhibition of this pathway with treatment. These results were also validated by IHC, insofar as the mTOR pathway downstream marker pS6 revealed a statistically significant decrease in IRS. The decrease in Ki-67 suggests that this inhibition of the mTOR pathway was associated with decreased tumor

Published by Bioscientifica Ltd 

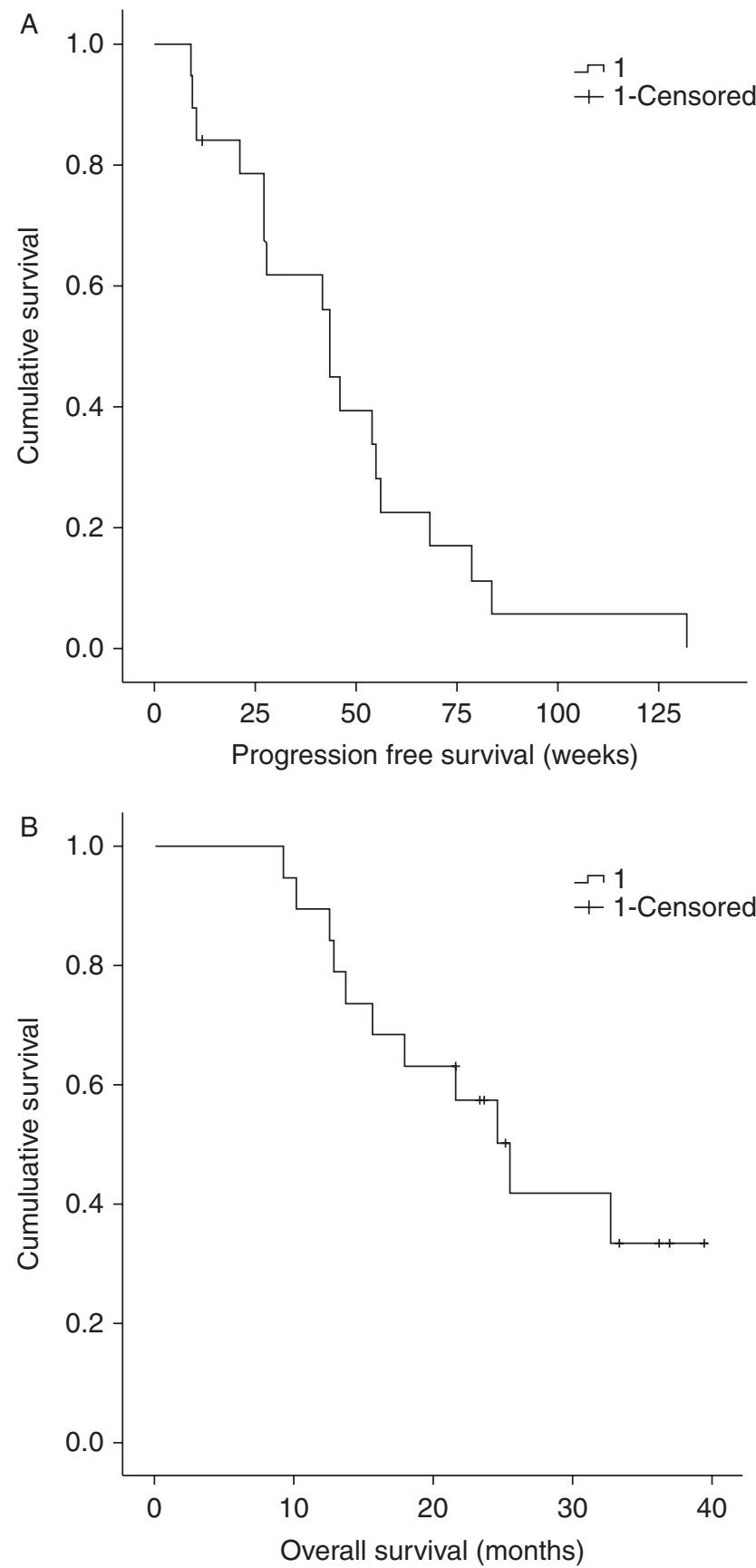

Figure 4

Progression-free survival (in weeks) (A) and overall survival (in months) (B).

proliferation between the initiation of therapy and the time of biopsy at the beginning of cycle 2 . However, this conflicts with the fact that only one patient had a partial response. Interestingly, that patient was the only one who did not require dose reductions and completed the study per protocol. This raises the possibility of a lack of adequate target inhibition because of dose reductions following this time point. The exploratory analyses aimed at identifying predictive baseline proteomic markers or PD changes. These are presented in the Supplementary Data (see section on supplementary data given at the end of this article). After adjusting for multiple testing, the marker that most strongly correlated with tumor shrinkage was the baseline estrogen receptor ER $\alpha$ expression (Supplementary Table 2). Recent work by our group has also suggested that estrogen-induced genes and progesterone receptors are up-regulated in indolent NETs as compared to more aggressive ones (Estrella et al. 2014a,b). These findings warrant further evaluation of estrogen and progesterone receptors and pathways as prognostic biomarkers in NETs.

Activated AKT is a key effector of the IGF and PI3K pathways via the phosphorylation of a vast number of proteins that affect multiple cellular responses, including metabolism. The net effect of these changes is increased glucose utilization and metabolism (Alayev \& Holz 2013). As an exploratory objective, we measured changes in indirect calorimetry from baseline to cycle 2 day 1 in 14 patients to evaluate the effect of inhibition of these pathways. We found a statistically significant decrease in basal oxygen consumption and metabolic rate. Although these findings are preliminary, the observed reduction in oxygen consumption is very intriguing. An ongoing trial (NCT01561404) is currently evaluating the positive impact of reduced oxygen consumption on physical exercise in patients who are being treated with mTOR inhibitors after renal transplant, and that trial may further validate the present findings.

The evaluation of antitumor activity as measured by response rate and PFS was a secondary objective of the present study. A major limitation of the evaluation of novel agents in NETs is that they often cause disease stabilization without true responses per RECIST criteria. Indeed, in the phase III trials that led to the approval of sunitinib and everolimus in pNETs, although radiographic responses were modest, there was a significant improvement in PFS as compared to the placebo arms (Raymond et al. 2011, Yao et al. 2011), which raised the question of whether other modalities for measuring response, such as modified RECIST, may be more suitable for NET. In the present study, given the small cohort size, definite conclusions regarding the activity of the combination are difficult to make. However, only one confirmed partial response per RECIST was noted. As discussed earlier in the present report, this could be related to a lack of adequate dosing of the combination. In this context, recent attempts at evaluating IGF1R

Published by Bioscientifica Ltd. 
MABs in NETs that have yielded disappointing results should also be noted (Reidy-Lagunes et al. 2012, Strosberg et al. 2013).

There are several potential explanations for this discrepancy between promising preclinical results and a lack of efficacy in clinical trials, including: i) a lack of biomarkers: it is conceivable that only a subset of NETs respond to these agents, and further work is required to identify these putative subsets (Gualberto et al. 2010, Yee 2012). ii) Alternative signaling pathways: the treatment of RIP-Tag2 mice with cixutumumab failed to have a significant impact on pNET growth or invasiveness despite a reduction in IGF1R levels. This was demonstrated to be the result of signaling through the insulin receptor (Ulanet et al. 2010). Resistance to IGF1R blockade may be also be regulated by targets such as EGFR, Her2, or the overexpression of IGFR pathway components (Haluska et al. 2008, Garofalo et al. 2011). iii) Heterogeneity of patient populations: accumulating evidence points to differences in genetic and clinical profiles of NETs from different sites. Therefore, future studies should be designed to accrue homogenous subsets of NET patients through international collaboration to generate more meaningful conclusions. iv) Finally, these discrepancies between preclinical and clinical results may reinforce the inherent limitations of the reproducibility and translation of preclinical data (Begley \& Ellis 2012), especially in a tumor type such as NET, which lacks robust preclinical models.

In summary, the present phase I trial established the RP2D of the regimen to be $10 \mathrm{mg} / \mathrm{kg}$ cixutumumab, $10 \mathrm{mg}$ everolimus p.o. daily, and $20 \mathrm{mg}$ octreotide LAR i.m. every 21 days. However, long-term tolerability without dose reductions may be difficult. A clear signal of benefit for anti-IGF1R therapy was not observed, which is in line with prior clinical trials in this patient population. Further work is required to elucidate the potential predictive biomarkers of and the mechanism of resistance of this combination in NETs before a phase II trial can be launched.

\section{Supplementary data}

This is linked to the online version of the paper at http://dx.doi.org/10.1530/ ERC-15-0002.

\section{Declaration of interest}

J Yao: paid consultant, Novartis; research funding, Novartis. F MericBernstam: paid consultant, Novartis.

\section{Funding}

Support was provided by the Sheikh Khalifa Al Nahyan Ben Zayed Institute for Personalized Cancer Therapy (grant number 5U01CA062461 to J Yao), the NCATS Center for Clinical and Translational Sciences (grant number UL1 TR000371), the M.D. Anderson Cancer Center Support (grant number P30 CA016672), and Novartis.

\section{References}

Akcakanat A, Sahin A, Shaye AN, Velasco MA \& Meric-Bernstam F 2008 Comparison of Akt/mTOR signaling in primary breast tumors and matched distant metastases. Cancer 112 2352-2358. (doi:10.1002/ cncr.23456)

Alayev A \& Holz MK 2013 mTOR signaling for biological control and cancer. Journal of Cellular Physiology 228 1658-1664. (doi:10.1002/ jcp.24351)

Begley CG \& Ellis LM 2012 Drug development: raise standards for preclinical cancer research. Nature 483 531-533. (doi:10.1038/ 483531a)

Christofori G, Naik P \& Hanahan D 1995 Deregulation of both imprinted and expressed alleles of the insulin-like growth factor 2 gene during $\beta$-cell tumorigenesis. Nature Genetics 10 196-201. (doi:10.1038/ ng0695-196)

Estrella JS, Broaddus RR, Mathews A, Milton DR, Yao JC, Wang H \& Rashid A 2014a Progesterone receptor and PTEN expression predict survival in patients with low- and intermediate-grade pancreatic neuroendocrine tumors. Archives of Pathology \& Laboratory Medicine 138 1027-1036. (doi:10.5858/arpa.2013-0195-OA)

Estrella JS, Ma LT, Milton DR, Yao JC, Wang H, Rashid A \& Broaddus RR $2014 b$ Expression of estrogen-induced genes and estrogen receptor $\beta$ in pancreatic neuroendocrine tumors: implications for targeted therapy. Pancreas 43 996-1002. (doi:10.1097/MPA. 0000000000000203

Garofalo C, Manara MC, Nicoletti G, Marino MT, Lollini PL, Astolfi A, Pandini G, Lopez-Guerrero JA, Schaefer KL, Belfiore A et al. 2011 Efficacy of and resistance to anti-IGF-1R therapies in Ewing's sarcoma is dependent on insulin receptor signaling. Oncogene 30 2730-2740. (doi:10.1038/onc.2010.640)

Gualberto A, Dolled-Filhart M, Gustavson M, Christiansen J, Wang YF, Hixon ML, Reynolds J, McDonald S, Ang A, Rimm DL et al. 2010 Molecular analysis of non-small cell lung cancer identifies subsets with different sensitivity to insulin-like growth factor I receptor inhibition. Clinical Cancer Research 16 4654-4665. (doi:10.1158/1078-0432.CCR10-0089)

Haluska P, Carboni JM, TenEyck C, Attar RM, Hou X, Yu C, Sagar M, Wong TW, Gottardis MM \& Erlichman C 2008 HER receptor signaling confers resistance to the insulin-like growth factor-I receptor inhibitor, BMS-536924. Molecular Cancer Therapeutics 7 2589-2598. (doi:10.1158/ 1535-7163.MCT-08-0493)

Hennessy BT, Lu Y, Gonzalez-Angulo AM, Carey MS, Myhre S, Ju Z, Davies MA, Liu W, Coombes K, Meric-Bernstam F et al. 2010 A technical assessment of the utility of reverse phase protein arrays for the study of the functional proteome in non-microdissected human breast cancers. Clinical Proteomics 6 129-151. (doi:10.1007/s12014-010-9055-y)

Hopfner M, Baradari V, Huether A, Schofl C \& Scherubl H 2006 The insulin-like growth factor receptor 1 is a promising target for novel treatment approaches in neuroendocrine gastrointestinal tumours. Endocrine-Related Cancer 13 135-149. (doi:10.1677/erc.1.01090)

Iglewicz B \& Hoaglin DC 1993 In How to Detect and Handle Outliers (The ASQC Basic Reference in Quality Control). p16. Milwaukee, WI, USA: American Society for Quality Control.

Jiao Y, Shi C, Edil BH, de Wilde RF, Klimstra DS, Maitra A, Schulick RD, Tang LH, Wolfgang CL, Choti MA et al. 2011 DAXX/ATRX, MEN1, 
and MTOR pathway genes are frequently altered in pancreatic neuroendocrine tumors. Science 331 1199-1203. (doi:10.1126/science. 1200609)

Meric-Bernstam F \& Gonzalez-Angulo AM 2009 Targeting the mTOR signaling network for cancer therapy. Journal of Clinical Oncology $\mathbf{2 7}$ 2278-2287. (doi:10.1200/JCO.2008.20.0766)

Meric-Bernstam F, Akcakanat A, Chen H, Do KA, Sangai T, Adkins F, Gonzalez-Angulo AM, Rashid A, Crosby K, Dong M et al. 2012 PIK3CA/PTEN mutations and Akt activation as markers of sensitivity to allosteric mTOR inhibitors. Clinical Cancer Research 18 1777-1789. (doi:10.1158/1078-0432.CCR-11-2123)

Meric-Bernstam F, Akcakanat A, Chen H, Sahin A, Tarco E, Carkaci S, Adrada BE, Singh G, Do KA, Garces ZM et al. 2014 Influence of biospecimen variables on proteomic biomarkers in breast cancer. Clinical Cancer Research 20 3870-3883. (doi:10.1158/1078-0432. CCR-13-1507)

O'Reilly KE, Rojo F, She QB, Solit D, Mills GB, Smith D, Lane H, Hofmann F, Hicklin DJ, Ludwig DL et al. 2006 mTOR inhibition induces upstream receptor tyrosine kinase signaling and activates Akt. Cancer Research 66 1500-1508. (doi:10.1158/0008-5472.CAN-05-2925)

Pavel ME, Hainsworth JD, Baudin E, Peeters M, Horsch D, Winkler RE, Klimovsky J, Lebwohl D, Jehl V, Wolin EM et al. 2011 Everolimus plus octreotide long-acting repeatable for the treatment of advanced neuroendocrine tumours associated with carcinoid syndrome (RADIANT-2): a randomised, placebo-controlled, phase 3 study. Lancet 378 2005-2012. (doi:10.1016/S0140-6736(11)61742-X)

Raymond E, Dahan L, Raoul JL, Bang YJ, Borbath I, Lombard-Bohas C, Valle J, Metrakos P, Smith D, Vinik A et al. 2011 Sunitinib malate for the treatment of pancreatic neuroendocrine tumors. New England Journal of Medicine 364 501-513. (doi:10.1056/NEJMoa1003825)

Reidy-Lagunes DL, Vakiani E, Segal MF, Hollywood EM, Tang LH, Solit DB, Pietanza MC, Capanu M \& Saltz LB 2012 A phase 2 study of the insulin-like growth factor-1 receptor inhibitor MK-0646 in patients with metastatic, well-differentiated neuroendocrine tumors. Cancer 118 4795-4800. (doi:10.1002/cncr.27459)

Strosberg JR, Chan JA, Ryan DP, Meyerhardt JA, Fuchs CS, Abrams T, Regan E, Brady R, Weber J, Campos T et al. 2013 A multi-institutional, phase II open-label study of ganitumab (AMG 479) in advanced carcinoid and pancreatic neuroendocrine tumors. Endocrine-Related Cancer 20 383-390. (doi:10.1530/ERC-12-0390)

Therasse P, Arbuck SG, Eisenhauer EA, Wanders J, Kaplan RS, Rubinstein L, Verweij J, Van Glabbeke M, van Oosterom AT, Christian MC et al. 2000 New guidelines to evaluate the response to treatment in solid tumors. Journal of the National Cancer Institute 92 205-216. (doi:10.1093/jnci/92. 3.205)

Thimmaiah KN, Easton J, Huang S, Veverka KA, Germain GS, Harwood FC \& Houghton PJ 2003 Insulin-like growth factor I-mediated protection from rapamycin-induced apoptosis is independent of Ras-Erk1-Erk2 and phosphatidylinositol 3'-kinase-Akt signaling pathways. Cancer Research 63 364-374.

Ulanet DB, Ludwig DL, Kahn CR \& Hanahan D 2010 Insulin receptor functionally enhances multistage tumor progression and conveys intrinsic resistance to IGF-1R targeted therapy. PNAS $\mathbf{1 0 7}$ 10791-10798. (doi:10.1073/pnas.0914076107)

von Wichert G, Jehle PM, Hoeflich A, Koschnick S, Dralle H, Wolf E, Wiedenmann B, Boehm BO, Adler G \& Seufferlein T 2000 Insulin-like growth factor-I is an autocrine regulator of chromogranin A secretion and growth in human neuroendocrine tumor cells. Cancer Research 60 4573-4581.

Wulbrand U, Remmert G, Zofel P, Wied M, Arnold R \& Fehmann HC 2000 mRNA expression patterns of insulin-like growth factor system components in human neuroendocrine tumours. European Journal of Clinical Investigation 30 729-739. (doi:10.1046/j.1365-2362.2000. 00700.x)

Yao JC, Hassan M, Phan A, Dagohoy C, Leary C, Mares JE, Abdalla EK, Fleming JB, Vauthey JN, Rashid A et al. 2008 One hundred years after "carcinoid": epidemiology of and prognostic factors for neuroendocrine tumors in 35,825 cases in the United States. Journal of Clinical Oncology 26 3063-3072. (doi:10.1200/JCO.2007.15.4377)

Yao JC, Shah MH, Ito T, Bohas CL, Wolin EM, Van Cutsem E, Hobday TJ, Okusaka T, Capdevila J, de Vries EG et al. 2011 Everolimus for advanced pancreatic neuroendocrine tumors. New England Journal of Medicine 364 514-523. (doi:10.1056/NEJMoa1009290)

Yee D 2012 Insulin-like growth factor receptor inhibitors: baby or the bathwater? Journal of the National Cancer Institute 104 975-981. (doi:10.1093/jnci/djs258)

Received in final form 20 March 2015

Accepted 21 April 2015

Made available online as an Accepted Preprint

21 April 2015 http://erc.endocrinology-journals.org

DOI: $10.1530 / E R C-15-0002$
(C) 2015 Society for Endocrinology Printed in Great Britain 